\title{
Cool gas and dust in M 33: Results from the HERschel M 33 Extended Survey (HERM33ES) ${ }^{\star}$
}

\author{
J. Braine ${ }^{1}$, P. Gratier ${ }^{1}$, C. Kramer ${ }^{2}$, E. M. Xilouris ${ }^{3}$, E. Rosolowsky ${ }^{4}$, C. Buchbender ${ }^{2}$, M. Boquien ${ }^{5}$, D. Calzetti ${ }^{5}$, \\ G. Quintana-Lacaci ${ }^{2}$, F. Tabatabaei ${ }^{6}$, S. Verley ${ }^{7}$, F. Israel ${ }^{8}$, F. van der Tak ${ }^{9}$, S. Aalto ${ }^{10}$, F. Combes ${ }^{11}$, \\ S. Garcia-Burillo ${ }^{12}$, M. Gonzalez ${ }^{2}$, C. Henkel ${ }^{6}$, B. Koribalski ${ }^{13}$, B. Mookerjea ${ }^{14}$, M. Roellig ${ }^{15}$, K. F. Schuster ${ }^{16}$, \\ M. Relaño ${ }^{17}$, F. Bertoldi ${ }^{18}$, P. van der Werf ${ }^{8}$, and M. Wiedner ${ }^{11}$ \\ ${ }^{1}$ Laboratoire d'Astrophysique de Bordeaux, Université Bordeaux 1, Observatoire de Bordeaux, OASU, UMR 5804, CNRS/INSU, \\ BP 89, 33270 Floirac, France \\ e-mail: Braine@obs.u-bordeaux1.fr \\ 2 Instituto Radioastronomia Milimetrica (IRAM), Av. Divina Pastora 7, Nucleo Central, 18012 Granada, Spain \\ 3 Institute of Astronomy and Astrophysics, National Observatory of Athens, P. Penteli, 15236 Athens, Greece \\ ${ }^{4}$ University of British Columbia, Okanagan, 3333 University Way, Kelowna BC V1V 1 V7 Canada \\ 5 Department of Astronomy, University of Massachusetts, Amherst, MA 01003, USA \\ ${ }^{6}$ Max-Planck-Institut für Radioastronomie (MPIfR), Auf dem Hügel 69, 53121 Bonn, Germany, Argelander Institut fr Astronomie. \\ Auf dem Hügel 71, 53121 Bonn, Germany \\ 7 Dept. Física Teórica y del Cosmos, Universidad de Granada, Spain \\ 8 Leiden Observatory, Leiden University, PO Box 9513, 2300 RA Leiden, The Netherlands \\ 9 SRON Netherlands Institute for Space Research, Landleven 12, 9747 AD Groningen, The Netherlands \\ 10 Onsala Space Observatory, Chalmers University of Technology, 43992 Onsala, Sweden \\ 11 Observatoire de Paris, LERMA, 61 Av. de l'Observatoire, 75014 Paris, France \\ 12 Observatorio Astronómico Nacional (OAN) - Observatorio de Madrid, Alfonso XII 3, 28014 Madrid, Spain \\ 13 Australia Telescope National Facility, CSIRO, PO Box 76, Epping, NSW 1710, Australia \\ 14 Department of Astronomy \& Astrophysics, Tata Institute of Fundamental Research, Homi Bhabha Road, 400005 Mumbai, India \\ 15 KOSMA, I. Physikalisches Institut, Universität zu Köln, Zülpicher Straße 77, 50937 Köln, Germany \\ 16 IRAM, 300 rue de la Piscine, 38406 St. Martin d'Hères, France \\ 17 Institute of Astronomy, University of Cambridge, Madingley Road, Cambridge CB3 0HA, UK \\ 18 Argelander Institut fr Astronomie. Auf dem Hügel 71, 53121 Bonn, Germany
}

Received 30 March 2010 / Accepted 11 May 2010

\section{ABSTRACT}

\begin{abstract}
We present an analysis of the first space-based far-IR-submm observations of M33, which measure the emission from the cool dust and resolve the giant molecular cloud complexes. With roughly half-solar abundances, M 33 is a first step towards young low-metallicity galaxies where the submm may be able to provide an alternative to $\mathrm{CO}$ mapping to measure their $\mathrm{H}_{2}$ content. In this Letter, we measure the dust emission cross-section $\sigma$ using SPIRE and recent CO and H I observations; a variation in $\sigma$ is present from a nearsolar neighborhood cross-section to about half-solar with the maximum being south of the nucleus. Calculating the total $\mathrm{H}$ column density from the measured dust temperature and cross-section, and then subtracting the H I column, yields a morphology similar to that observed in $\mathrm{CO}$. The $\mathrm{H}_{2} / \mathrm{H}$ I mass ratio decreases from about unity to well below $10 \%$ and is about $15 \%$ averaged over the optical disk. The single most important observation to reduce the potentially large systematic errors is to complete the CO mapping of M 33 .
\end{abstract}

Key words. galaxies: individual: M 33 - Local Group - galaxies: evolution - galaxies: ISM - ISM: clouds - stars: formation

\section{Introduction, data, and dust temperature}

Understanding star formation requires studying the interplay between the phases of the interstellar medium (ISM). Dust processes most of the energy transiting the ISM, but the cool dust component, although representing the vast majority of the dust mass, is difficult to observe from the ground. Herschel SPIRE observations (Pilbratt 2010; Griffin 2010) are the first

* Herschel is an ESA space observatory with science instruments provided by European-led Principal Investigator consortia and with important participation from NASA. space-based $250-500 \mu \mathrm{m}$ data and as such provide a unique occasion to put together a global picture of the cool gas and dust in M33. In particular, we compare the morphology of the FarIR emission and that of the gas as determined from $\mathrm{CO}$ and H I measurements and attempt to measure how the dust crosssection varies in M33. A longer term goal is to be able to use the dust emission to constrain the variation of the $N\left(\mathrm{H}_{2}\right) / I_{\mathrm{CO}}$ factor within M 33 and elsewhere.

This Letter is one of a series on the HERM33ES project on the ISM of the Local Group galaxy M 33, an overview of which is given in Kramer et al. (2010), hereafter K10. For consistency with the other M33 papers in this volume, we adopt a 


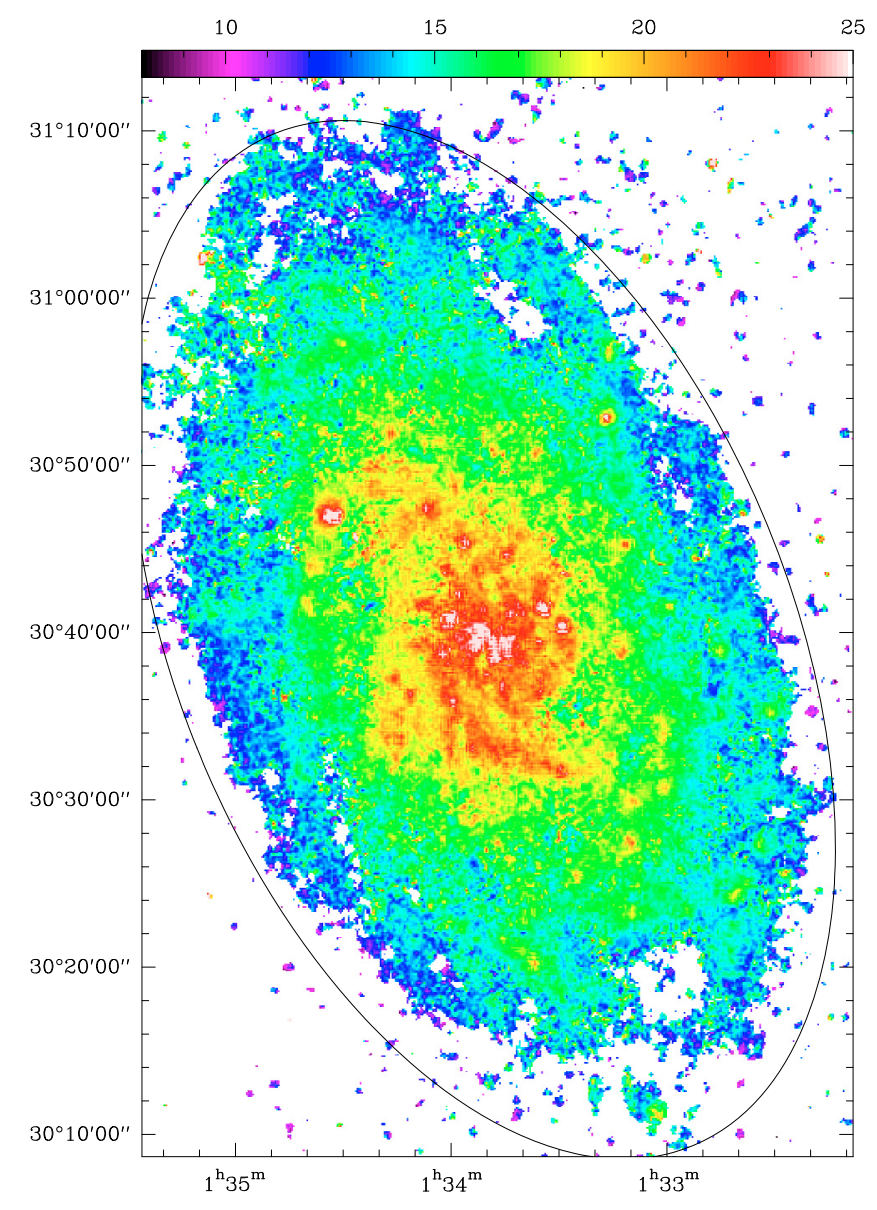

Fig. 1. Dust color temperature map of M 33 calculated from the SPIRE 250 and $350 \mu \mathrm{m}$ flux ratio with $\beta=2$ at the $\sim 25^{\prime \prime}$ resolution of the $350 \mu \mathrm{m}$ data. Coordinates are $\mathrm{J} 2000$, the temperature scale is shown at the top in Kelvin, and the ellipse shows the $8 \mathrm{kpc}$ radius, slightly beyond $\mathrm{R}_{25}$.

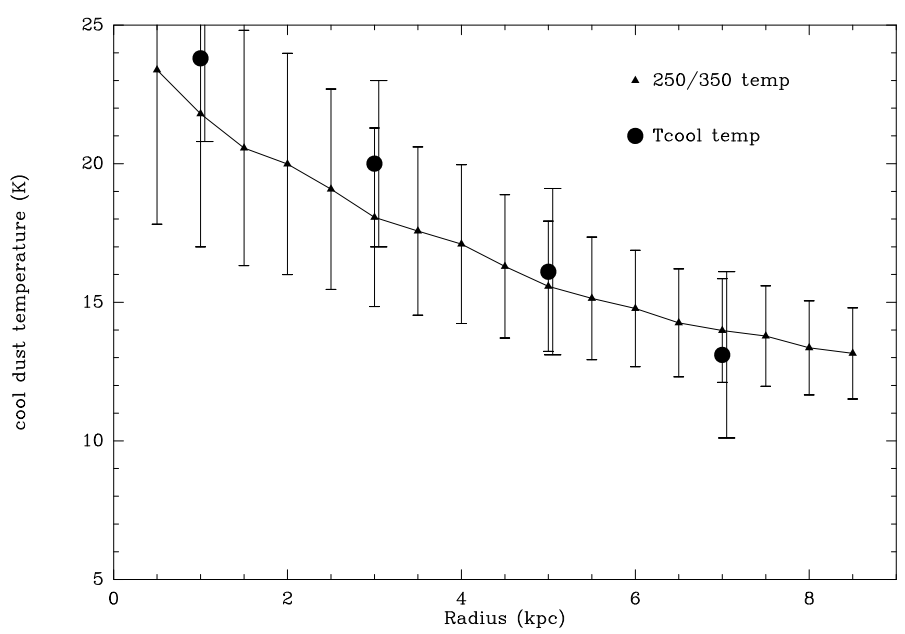

Fig. 2. Radial distribution of the average dust temperature in the stellar disk of M 33. The solid line joining the triangles shows the $250 / 350 \mu \mathrm{m}$ color temperature and for comparison we show the cool dust temperature (large dots) obtained by K10 in their preferred two-component model with $\beta=1.5$. The error bars on the triangles indicate a $15 \%$ calibration uncertainty in the $250 / 350 \mu$ m ratio converted to temperature.

distance of $D=840 \mathrm{kpc}$ for M33 (i.e., $25 \operatorname{arcsec}=100 \mathrm{pc}$ ) and orientation parameters of $P A=22.5$ degrees and $i=56^{\circ}$. We use the recent HERM33ES SPIRE observations at 250, 350, and $500 \mu \mathrm{m}$ combined with $\mathrm{CO}(2-1)$ observations from the
M33CO@IRAM project, as a tracer of the molecular component, and a high-resolution mosaic of VLA HI data (both from Gratier et al. 2010). The SPIRE data were first processed as described in $\mathrm{K} 10$ and then converted from Jy/beam to brightness units (MJy/sr). To estimate the dust temperature, the $250 \mu \mathrm{m}$ data were convolved to the $350 \mu \mathrm{m}$ beamsize $\left(\sim 25^{\prime \prime}\right)$ and, assuming a single temperature grey body with an emissivity $\propto \nu^{\beta}$ with $\beta=2$, a temperature was derived from the flux ratio. At the temperatures of the cool component seen in M 33, Dupac et al. (2003) find $\beta \approx 2$. To minimize the effect of the uncertainty in $\beta$, we chose to use adjacent bands to estimate temperature. The $250 / 350 \mu \mathrm{m}$ ratio provides more accurate temperatures than the $350 / 500$ micron ratio even for temperatures below $10 \mathrm{~K}$. A $15 \%$ variation (or uncertainty) in the $250 / 350 \mu \mathrm{m}$ ratios corresponds to a temperature change of $0.9,2.1$, and $4.1 \mathrm{~K}$ at temperatures of 10,15 , and $20 \mathrm{~K}$ but the same uncertainty in the $350 / 500 \mu \mathrm{m}$ ratios yields $1.3,3.2$, and $6.3 \mathrm{~K}$ errors for the same dust temperatures. A further advantage is that we obtain the dust temperature at a resolution typical of giant molecular clouds (GMC), $\sim 100$ pc.

Ideally, a multi-component fit would be used but this requires high $\mathrm{S} / \mathrm{N}$ data at many wavelengths over the whole disk. By assuming that the cool dust component dominates the emission beyond $250 \mu \mathrm{m}$, we could calculate the dust temperature out to the optical radius of $\mathrm{M} 33$. The resulting temperature map is shown in Fig. 1. Figure 2 shows a comparison with the temperature of the cool component of the preferred two-component (warm plus cool dust) model fit to data between $24 \mu \mathrm{m}$ and $500 \mu \mathrm{m}$ from K10. The two-component model uses $\beta=1.5$ and the temperatures are higher out to $6 \mathrm{kpc}$. The temperatures in the radial bins have been estimated by averaging the temperatures rather than averaging the emission as in K10. The latter yields slightly higher temperatures because the dust is usually warmer where emission is strong (compare Fig. 1 with Fig 1. in K10). K10 calculate the dust column density using $\kappa=$ $0.4(v / 250 \mathrm{GHz})^{2} \mathrm{~cm}^{2} \mathrm{~g}^{-1}$ of dust, which is equivalent to a dust cross-section per H-atom of $\sigma=1.1 \times 10^{-25}(\lambda / 250 \mu \mathrm{m})^{-2} \mathrm{~cm}^{2}$ for a hydrogen gas-to-dust mass ratio of 140 . In the following we use the SPIRE 250/350 $\mu \mathrm{m}$ color temperature because the data cover a greater area and agree well with the cool dust temperature of the two-component fit, showing the domination of the cool dust at these wavelengths. No correction for line contamination was subtracted from the SPIRE data. At these frequencies, the CO lines contribute very little to the continuum flux, unlike at $1.3 \mathrm{~mm}$ or $850 \mu \mathrm{m}$ (e.g. Braine et al. 1997).

Figure 3 shows the SPIRE $250 \mu \mathrm{m}$ emission with the $\mathrm{CO}(2-$ 1 ) emission as contours at $25^{\prime \prime}$ resolution to show how closely the $\mathrm{CO}$ emission follows the dust emission peaks. Gratier et al. (2010) show a detailed comparison of the CO and H I emission with star formation tracers such as $\mathrm{H} \alpha$ and Spitzer 8 and $24 \mu \mathrm{m}$ maps. The $250 \mu \mathrm{m}$-bright regions are detected in $\mathrm{CO}$ and the general morphology of the cool dust emission is seen in the H I image.

\section{Dust cross-section}

In order to estimate the total gas mass of a galaxy from its dust emission, it is first necessary to measure the dust emission crosssection per $\mathrm{H}$-atom $\sigma$. We do this by selecting regions with $\mathrm{HI}$ emission and well-constrained dust temperatures but with little or no $\mathrm{CO}$ emission (in order to avoid the assumption of a $N\left(\mathrm{H}_{2}\right) / I_{\mathrm{CO}}$ value and $\mathrm{CO}$ line ratio). Thereby, we can relate the observed neutral hydrogen column density, $N_{\mathrm{HI}}$, to the dust optical depth, $\tau=\sigma N_{\mathrm{H}}=S_{v} / B_{v, T}$, and infer the cross section $\sigma$. 
$10^{1}$

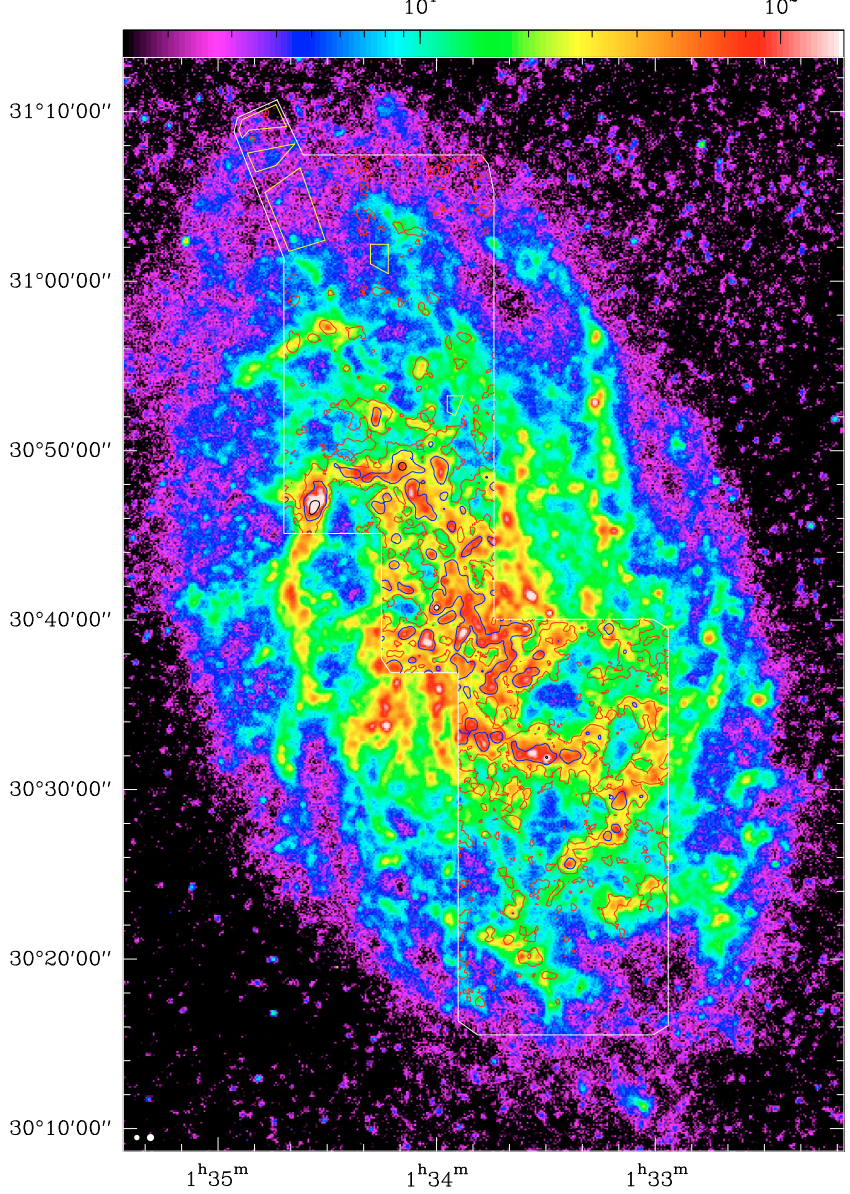

Table 1. Dust cross-section $\sigma$ at $250 \mu \mathrm{m}$ as a function of radius in $\mathrm{M} 33$, expressed in units of $1.1 \times 10^{-25} \mathrm{~cm}^{2}$ per $\mathrm{H}$-atom.

\begin{tabular}{lrrrrrr}
\hline \hline$r(\mathrm{kpc})$ & 4 & 5 & 5.5 & 6 & 7 & 7.5 \\
\hline Polygons & 1.8 & 1.02 &.. & 1.07 & 0.66 & 0.50 \\
histo- $N$ & 0.65 &.. & 0.54 &.. &.. & 0.48 \\
histo- $S$ & 0.92 &.. & 0.95 & 0.69 &.. &.. \\
Model & 0.8 &.. & 0.75 &.. & 0.66 & 0.5 \\
\hline
\end{tabular}

Notes. The polygons are shown in Fig. 3. The histogram method was applied separately in the north and south (lines 2 and 3), leading to the "Model" values used to estimate the total $\mathrm{H}$ column density to make Fig. 4.

for the areas without detected $\mathrm{CO}$ emission but with HI emission and a constrained dust temperature, yielding the lower values for $\sigma$, as shown in Table 1. Using this method, we find a north-south difference in M 33 at equivalent radii. The uncertainty in the histogram method is about \pm 0.1 ; although the distribution is sometimes broad, the peak value is well defined. The histograms of $\sigma$ values are clearly different in the north and south. The polygon method was difficult to use in the south due to the absence of large CO-free regions with $\mathrm{HI}$ and dust emission. An advantage of the histogram method is that it excludes the tail of high $\sigma$ which may be due to $\mathrm{H}_{2}$ without detected $\mathrm{CO}$ emission.

The intrinsic expectation is that because the oxygen abundance is about half solar, the dust cross-section should be as well (Draine et al. 2007), and that given the shallow abundance gradient in M 33 (Rosolowsky et al. 2007; Magrini et al. 2009), this should hold for the entire galaxy. However, it quickly became apparent that the dust cross-section per $\mathrm{H}$-atom varied over the galaxy, decreasing with radius by close to a factor 2 , with a higher abundance in the south - similar to the variation found by Magrini et al. (2010) from optical H II region and $\mathrm{PNe}$ abundance measurements. In the solar neighborhood, $\sigma$ is about $\sigma \approx 1.1 \times 10^{-25} \mathrm{~cm}^{2}$ per H-atom (Draine \& Lee 1984; Draine $\& \mathrm{Li} 2007)$ at $250 \mu \mathrm{m}$ and varying as $v^{2}$. If we apply a $\sigma$ of half this value to M 33 using the dust temperature map in Fig. 1, we obtain unrealistically high gas masses in the inner disk: $\sim 40 M_{\odot}$ $\mathrm{pc}^{-2}$ on average, whereas the $\mathrm{HI}$ is about $11 M_{\odot} \mathrm{pc}^{-2}$ and the $\mathrm{H}_{2}$ considerably less (Gratier et al. 2010). For a half-solar $\sigma$, the (cool) dust temperature would have to be about $30 \mathrm{~K}$, beyond any experimental uncertainties, so we conclude that $\sigma$ must be higher in the inner disk. In the outer disk, however, the H I column density (where no $\mathrm{CO}$ emission is present) is roughly equal to what is derived from the dust with $\sigma=0.5 \sigma_{\odot}$, suggesting that this value is appropriate for the outer disk.

\section{Total and molecular gas mass}

To really obtain an accurate gas mass from the cold dust emission data, the calibration uncertainties in the SPIRE bands must be reduced because the $15 \%$ uncertainty is not sufficient to distinguish between different solutions of $\beta$ and temperature. A second requirement is that the whole disk of M 33 must be observed in $\mathrm{CO}$ so that a well-determined variation of $\sigma$, based on the second technique, can be constructed. The H I data provide a reliable picture of the $\mathrm{HI}$ column density, so subtracting this from the total gas column density derived from the dust emission yields an estimate of the molecular gas mass which does not rely on $\mathrm{CO}$ emission. A further caveat is that $\sigma$ must not change from the atomic to molecular medium. Once the [C I] and [C II] lines have been measured, these uncertainties can be reduced by the additional constraint that the $\mathrm{C} / \mathrm{H}$ ratio (from the sum of the $\mathrm{C}$ 


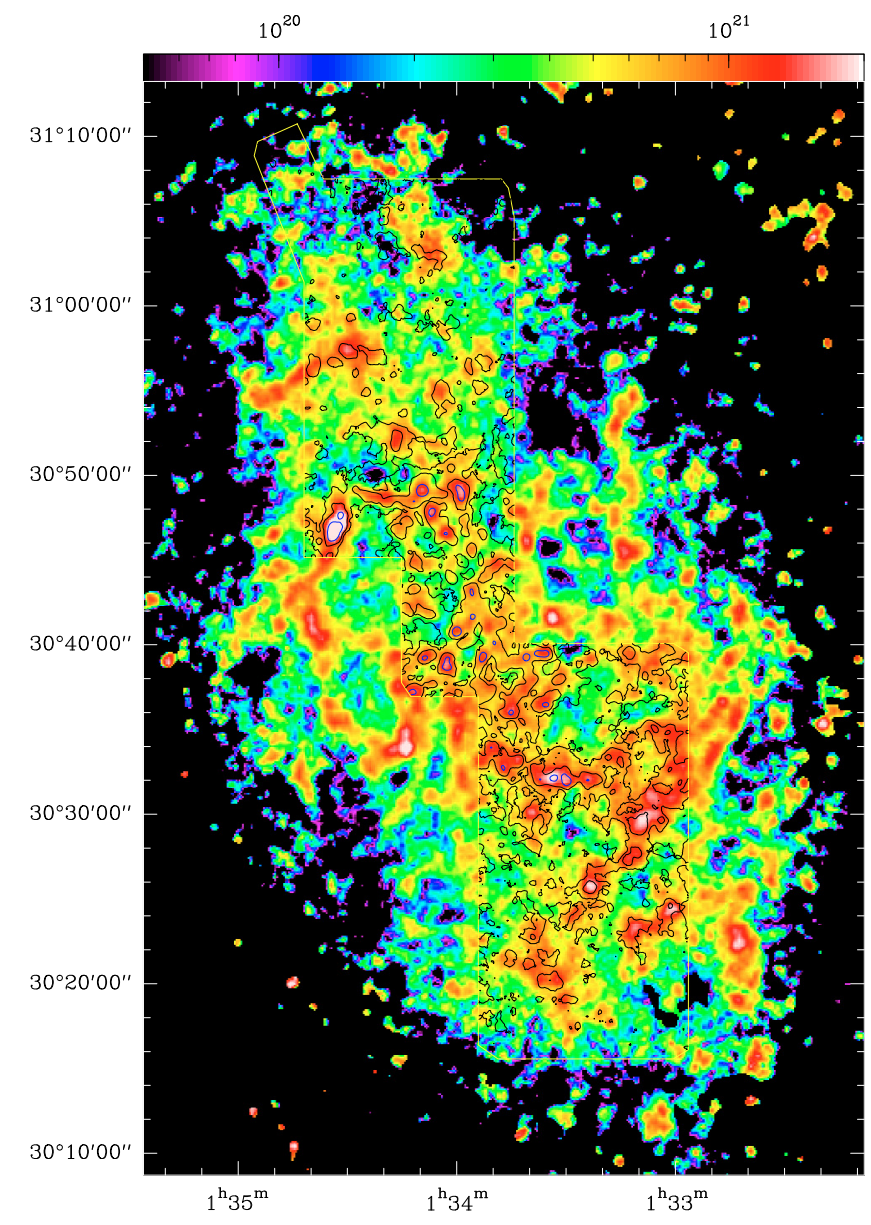

Fig. 4. $\mathrm{H}_{2}$ column density map estimated from the $500 \mu \mathrm{m}$ SPIRE map, $\beta=2$, the $250 / 350 \mu \mathrm{m}$ dust temperature as shown in Fig. 1, the H I data from Gratier et al. (2010), and the cross-sections given in Table 1. The column density scale is shown at the top in units of $\mathrm{H}_{2}$ molecules per $\mathrm{cm}^{2}$. CO contours at 0.4 and $1.6 \mathrm{~K} \mathrm{~km} \mathrm{~s}^{-1}$ (black) and $5 \mathrm{~K} \mathrm{~km} \mathrm{~s}^{-1}$ (blue) are superposed.

reservoirs) should be equal to the $\mathrm{C}$ abundance derived by other means.

Using the information currently available, we have used Table 1 (second method) to build an image of the dust crosssection $\sigma$, erring on the low- $\sigma$ side, as indicated in the last line of Table 1 . We then used this $\sigma$, scaled to $500 \mu \mathrm{m}$ with $\beta=2$, and the dust temperature (Fig. 1) to estimate the total gas mass from the $500 \mu \mathrm{m}$ data and then subtracted the H I column density to obtain the $\mathrm{H}_{2}$ column density using the interferometric H I maps from Gratier et al. (2010), which recover more than $90 \%$ of the flux found by Putman et al. (2009) using Arecibo. The missing $21 \mathrm{~cm}$ flux is expected to be located in the mid-toouter disk where the rotation curve is fairly flat. A morphological comparison of the dust-derived $\mathrm{H}_{2}$ column and the $\mathrm{CO}$ contours in Fig. 4 shows that while major regions were not missed by the $\mathrm{CO}$ observations, the dust-derived $\mathrm{H}_{2}$ map does not correlate perfectly with the $\mathrm{CO}$. For example, the strong $\mathrm{CO}$ peak at $\left(01^{h} 34^{\mathrm{m}} 09^{\mathrm{s}} .4,+30^{\circ} 49^{\prime} 06^{\prime \prime}\right)$ corresponds to a red (not white) region in Fig. 4; this may of course reflect a real variation in the $N\left(\mathrm{H}_{2}\right) / I_{\mathrm{CO}}$ ratio.

The data used in the scatter plot in Fig. 5 (see also Fig. 7 in Leroy et al. 2009) yield a formal fit with a $N\left(\mathrm{H}_{2}\right) / I_{\mathrm{CO}(2-1)}=3 \times$ $10^{20} \mathrm{H}_{2}$ per $\mathrm{K} \mathrm{km} \mathrm{s}^{-1}$ value, corresponding to a roughly galactic $N\left(\mathrm{H}_{2}\right) / I_{\mathrm{CO}(1-0)}=2.1 \times 10^{20} \mathrm{H}_{2}$ per $\mathrm{K} \mathrm{km} \mathrm{s}^{-1}$ for a line ratio $\mathrm{CO}\left(\frac{2-1}{1-0}\right)=0.7$. The inner disk $N\left(\mathrm{H}_{2}\right) / I_{\mathrm{CO}(2-1)}$ is lower and rises

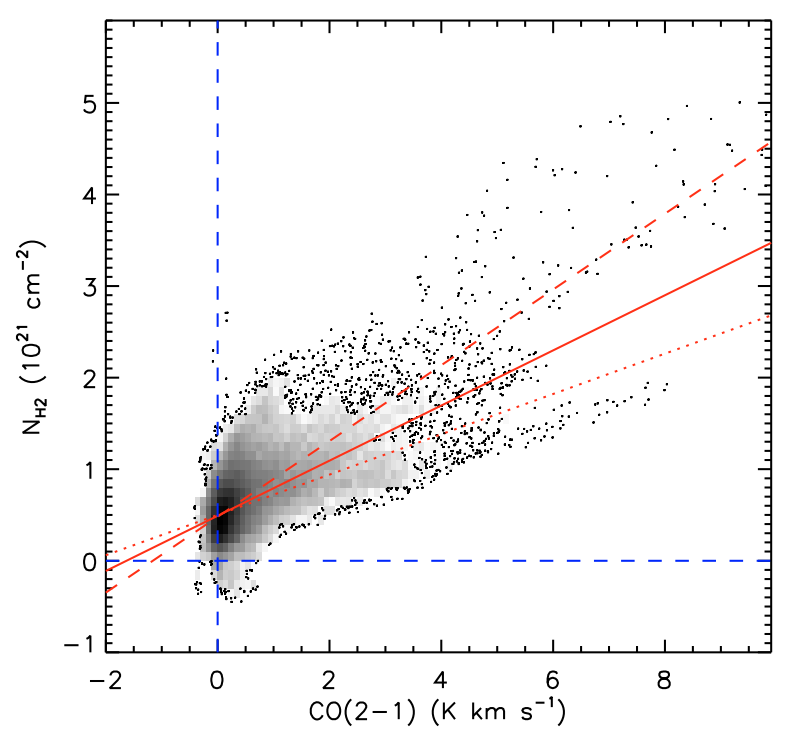

Fig. 5. Scatter plot of $\mathrm{H}_{2}$ column density as derived from the dust emission and the $\mathrm{CO}(2-1)$ intensity. The solid red line is a fit to the entire dataset and corresponds to a $N\left(\mathrm{H}_{2}\right) / I_{\mathrm{CO}(2-1)}$ value of $3 \times 10^{20} \mathrm{H}_{2}$ per $\mathrm{K} \mathrm{km} \mathrm{s}^{-1}$ with an offset as described in the text. A radial variation is present: fitting within $2 \mathrm{kpc}$ yields a $N\left(\mathrm{H}_{2}\right) / I_{\mathrm{CO}(2-1)}$ value of $2.2 \times 10^{20} \mathrm{H}_{2}$ per K km s$~^{-1}$ and $4.1 \times 10^{20} \mathrm{H}_{2}$ per $\mathrm{K} \mathrm{km} \mathrm{s}^{-1}$ beyond $2 \mathrm{kpc}$ (dotted and dashed lines, respectively).

to about $4 \times 10^{20} \mathrm{H}_{2}$ per $\mathrm{K} \mathrm{km} \mathrm{s}^{-1}$ beyond $2 \mathrm{kpc}$. The offset, in principle, implies that some low column density $\mathrm{H}_{2}$ is not seen in CO. Both values depend strongly on the details of the dust cross-section, illustrating the need for a complete map of M 33 in $\mathrm{CO}$.

The total hydrogen mass derived from the dust emission within $8 \mathrm{kpc}$ is estimated to be $1.63 \times 10^{9} M_{\odot}$, very similar to the more directly measured $\mathrm{H} \mathrm{I}+\mathrm{H}_{2}$ masses in Gratier et al. (2010). The dust cross-sections we derive suggest dust-to-gas mass ratios (including $\mathrm{He}$ ) ranging from $\sim 125$ in the inner $4 \mathrm{kpc}$ to 200 near $\mathrm{R}_{25}$ for Milky Way like dust ( $c f$. K10). Comparing the total dust-derived $\mathrm{H}_{2}$ mass with the $\mathrm{CO}$ luminosity (Gratier et al. 2010) yields a $N\left(\mathrm{H}_{2}\right) / I_{\mathrm{CO}}$ of about 1.5 times the Galactic $N\left(\mathrm{H}_{2}\right) / I_{\mathrm{CO}}$. Because the $\mathrm{H}$ I dominates, however, a small error in the total dust-derived gas mass translates into a large uncertainty in the dust-derived $\mathrm{H}_{2}$ mass. It is clearly necessary to obtain whole-galaxy measurements of $\sigma$ in $\mathrm{CO}$-free zones to refine these estimates.

\section{References}

Braine, J., Guélin, M., Dumke, M., et al. 1997, A\&A, 326, 963 Draine, B. T., \& Lee, H. M. 1984, ApJ, 285, 89

Draine, B. T., \& Li, A. 2007, ApJ, 657, 810

Draine, B. T., Dale, D. A., Bendo, G., et al. 2007, ApJ, 663, 866

Dupac, X., Bernard, J., Boudet, N., et al. 2003, A\&A, 404, L11

Gardan, E., Braine, J., Schuster, K. F., Brouillet, N., \& Sievers, A. 2007, A\&A, 473,91

Gratier, P., Braine, J., Rodriguez-Fernandez, N. J., et al. 2010, A\&A, accepted [arXiv: 1003.3222]

Griffin, M. J., et al. 2010, A\&A, 518, L3

Kramer, C., et al. 2010, A\&A, 518, L67

Leroy, A. K., Bolatto, A., Bot, C., et al. 2009, ApJ, 702, 352

Magrini, L., Stanghellini, L., Corbelli, E., Galli, D., \& Villaver, E. 2010, A\&A, 512,63

Magrini, L., Stanghellini, L., \& Villaver, E. 2009, ApJ, 696, 729

Pilbratt, G. L., et al. 2010, A\&A, 518, L1

Putman, M. E., Peek, J. E. G., Muratov, A., et al. 2009, ApJ, 703, 1486

Rosolowsky, E., Keto, E., Matsushita, S., \& Willner, S. P. 2007, ApJ, 661, 830 